\title{
A Pragmatic Business Approach to a Novel Aerial Radio Architecture, SIIARA
}

\author{
Purnima Lala Mehta, Ambuj Kumar and Ramjee Prasad \\ Department of Business Development and Technology, \\ School of Business and Social Sciences, Aarhus University, \\ Herning, Denmark \\ E-mail:purnima.lala@gmail.com; ambuj@btech.au.dk; ramjee@btech.au.dk
}

Received 10 January 2018; Accepted 15 February 2018;

Publication 20 April 2018

\begin{abstract}
The future wireless communications are expected to offer a variety of new technologies and services to the mass subscriber base. Innovative solutions for alternate mobile network infrastructure is likely to become a much-needed modification in the future to accommodate the challenges of ultra-dense HetNets related to dynamic service needs of mobility-driven mass network users. Previously, we have proposed a novel Aerial Radio Architecture, as an alternate network to the ground infrastructure to operate under network congestion scenarios. Until now, there has not been much business model innovation related to the mobile network infrastructure. The focus of our study is to insinuate business pragmatism to our proposed concepts of Aerial Radio Architectures and scrutinize from a business point of view. In this paper, we propose and discuss the need to shift from the classic business modelling to pragmatic business modelling through a Persuasive and Disruptive business technology approach. We aim to give an insight to the business perspective by illustrating multiple business case scenarios through the Business Model Cube with the goal of creating business and enhancing revenue growth rates to the network service operators.
\end{abstract}

Journal of Multi Business Model Innovation and Technology, Vol. 4_3, 215-240, River Publishers

doi: $10.13052 / \mathrm{jmbmit} 2245-456 \mathrm{X} .433$

This is an Open Access publication. (c) 2018 the Author(s). All rights reserved. 
Keywords: Network infrastructure expansion, mobility-driven mass users, Aerial Radio Architecture, Persuasive and Disruptive business approach, Business Model Cube.

\section{Introduction}

The future wireless communications are touted to bring both evolution and revolution in the ICT industry. Until now, research has reached an advanced stage to bring the $5 \mathrm{G}$ and beyond communication networks into existence. Studies and proposals on improving various aspects of a telecom generation network like upgraded mobile technologies, network infrastructure, spectrum, and energy management, etc. have been performed with the goal of satisfying the end user's experience. The future heterogeneous networks will combine the macro, micro, pico and femto cells to assuage the two most important aspects of a radio network service provisioning, Coverage, and Capacity. The practical deployment Ultra Dense Networks (UDN) formed of a number of large and small cells in a particular network area come with the issues of site costs (design, construction, and acquisition), stationing and spacing, and suitability of locations, especially in populated urban cities. The network will evolve from hot-spots (1-2 small cells per macro) to hot-zones (4-6 small cells per macro) to ultra-dense networks (10-12 small cells per macro), raising concerns for interference and mobility management for the users [1].

The widespread adoption of new technologies with inventive concepts of Internet of Things (IoT), Network Function Virtualization (NFV), Software Defined Networking (SDN), etc. amongst the huge and growing subscriber base, certainly requires the redesigning and rethinking the way in which the services that are delivered by the network operators and owners to the end users.

The network infrastructure is expected to be redesigned with upgradations of services, capabilities, architecture, interfaces, etc. [2]. Huawei in their white paper mention the requirement to a rethink, restructure, and redesign the of approaches to mobile network construction and expansion [3]. The limitations of the monolithic infrastructure in the current mobile networks can be addressed through solutions like adding new sites and improving spectral efficiency; there is indeed an urge to redesign the mobile network infrastructure [4]. User mobility and device-to-device connections being the necessary drivers for the change in the network design. 


\subsection{Background and Motivation}

In our previous works, we have proposed and investigated a Self-Itinerant Intelligent Radio Architecture (SIIARA) [5] to provide 'Service in Motion' demands at mobility-oriented and heavily accumulated user events [6], as shown in Figure 1. The SIIARA comprises of multiple Hovering Ad-hoc Networks (HANETs) [7], built up of airborne base stations connected into the formation of spatial ad-hoc networks above the congested network users.

The service delivery requirements of the mass users (especially mobilitydriven) are going to pose challenges to the existing traditional business models [3]. Any new solution to the technological market requires monetizing the new technology and services, with an all-time service delivery goal (with required QoS) to the mobility-driven end customers. This aspect is essential to the service operators as the new implementations depend upon huge investments that must result in creating a good business from the offered services to the investors and service operators.

The future communication networks are now required to be seen from the technical as well as from the business perspective. Our proposed concepts mentioned previously have given a framework of Aerial Radio Architecture, for targeting the dynamic service requirements of the mass moving users. The conceptual idea being the foundation of our study requires the actual product manufacturing for the practical deployment and to exploit its usability in real-time network congestion scenarios and 'On-Demand' service needs. It is important to validate a novel concept from the business and implementation perspective such that the mobile infrastructure investment in creating the prototype creates strong business opportunities result in the profitability to the service operators and other parties and investors involved. Through this paper, an attempt to quench the quest of understanding our previous findings from the business point of view is made.

\subsection{Problem Definition}

The core objective of the cellular network dimensioning and planning performed by the network service providers (more commonly known as 'operators') for a given network area is to make sure that the expected needs and demands of the subscribers are met in terms of economy, quality, availability, and accessibility. The service operators target the user distributions per subarea to determine the number of cell sites required. An effective cellular network planning must cope with the increasing number of mobile data devices with the aim of attaining a decent network performance. 
HANET based SIIARASub-architecture-Area 2

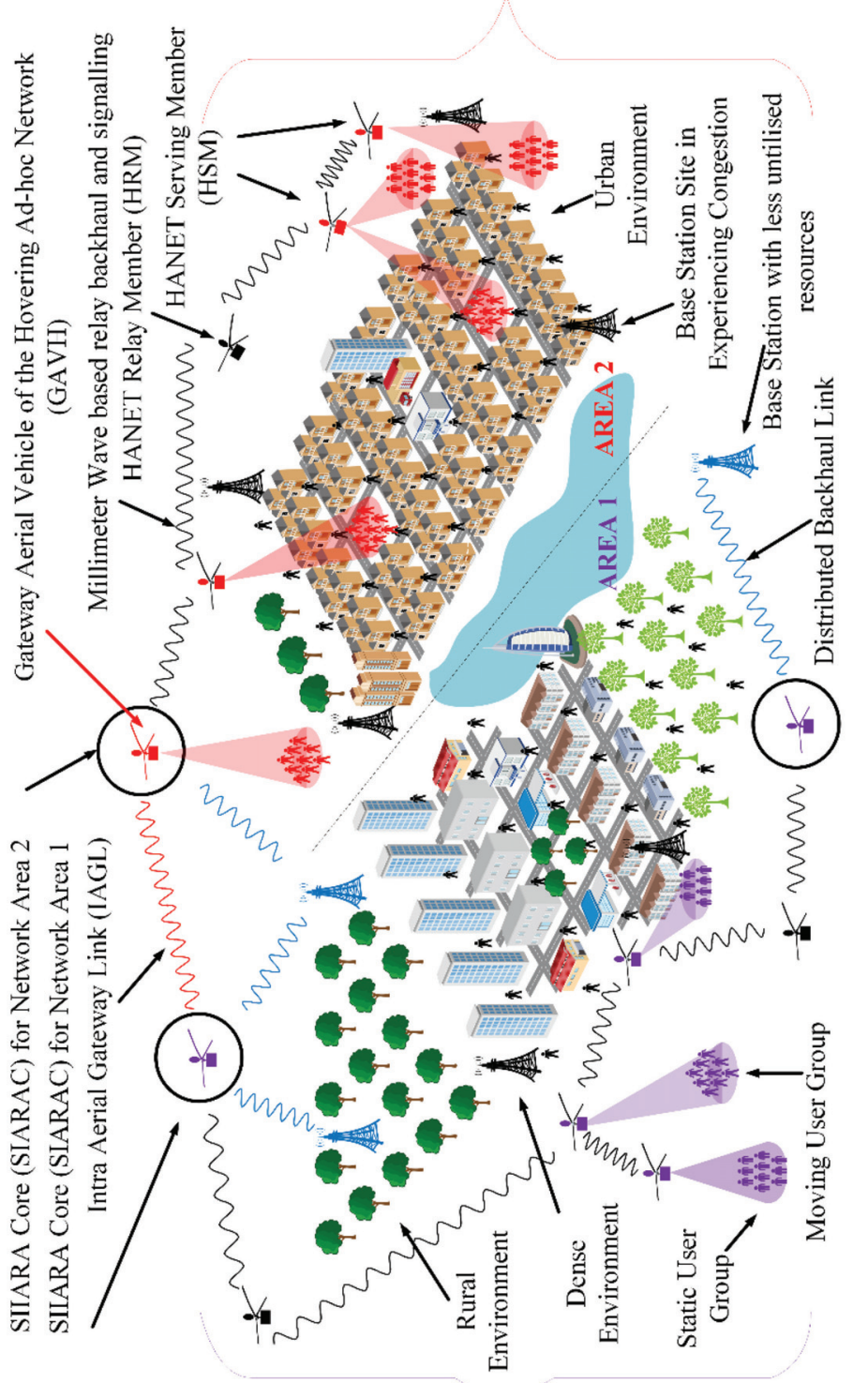

政

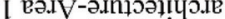

-qnS $\forall$ Y 
The user device mobility is one of the most challenging aspects of a wireless cellular system and must facilitate the users to be mobile and fulfil the demands for faster and better data services while on the move. It has been observed that user accumulation at any given network area, tends to overload the cell sites nearby. This network overloading is proportional to the number of devices present in the cell network area. Looking at the future network scenarios, the number of data demanding devices are going to increase aggressively, and the possibility of the network overloading will become worse. The network issues concerning the massed user mobility, also known as the Place Time Capacity (PTC) [6], might pose a serious challenge for the service operators to look upon in the near future. The idea of multi-tier Heterogeneous Networks (HetNets) and densifying the network through small cells have been into implementation in the fixed network deployment system. However, a number of mobile devices increasing is an ongoing process, and there will come a time when the small cell addition and reducing site to site distance will have their limitations. The network complexity concerning the placement issues and interference shall put unimaginable complications to the service operators for the future wireless communications. Alternate solutions like COWs/COLTs [8], moving cells [9], etc. have also been proposed, but the extra costs of deployment and inability to target and follow the users at all times will make these solutions insufficient and ineffective to deploy.

In this paper, we delve into providing an innovative approach that can satisfy this problem and propose an appropriate business approach that makes the deployment of our concept economic and reasonable.

The remainder of this article is organised as follows: Section 2 gives the Related Work, presenting the business model approaches that have been proposed by far. The discussion on the network paradigm shift is provided in Section 3. Section 4 discusses our proposed pragmatic business modelling approach. Finally, this paper is concluded in Section 5.

\section{Related Work}

The business model innovation for the future networks is a pivotal consideration. It is important to overcome the challenges of the traditional models and hence a need for business model innovation. There is always a scope of improvement, enhancement, and advancement of the currently used models. Owing to the fact that business processes and the environment are not static, the business models should be dynamic and must change with the environment or make the environment change [10]. 
As stated by the authors in [10], a need for continuous monitoring, evaluation and improvement of the business model is imperative to sustain the business advantage. Multiple definitions and perspectives have been given in proposing the business models for various fields. However, the first instance in perceiving a business model is a conceptual, rather than a financial model [11]. A good business model yields value propositions that are compelling to the customers, achieves advantageous cost and risk structures, and enables significant value capture by the business that generates and delivers products and services [11].

The future communication networks will create an ecosystem for both technical and business innovation with most of the network services relying on software [12] that require the infrastructure to be flexible and responsive. It is imperative to bring new industrial stakeholders (e.g., vertical industries, novel forms of service providers or infrastructure owners and vendors), to build the top network architectures and infrastructures for the $5 \mathrm{G}$ and beyond radio systems [12]. Certainly, the new business models to be implemented must support the future network infrastructure in a way to reinforce flexibility and programmability (advanced software embedded with Artificial Intelligence), apart from supporting a variety of services everywhere and user mobility conditions.

The innovation, proposals, and research into future telecom generation business models are still in the beginning phase. We do not see a clear picture of the research and implementation of potential revenue and business models by the telecom giants. The two internet giants Facebook and Google are testing their proposals of 'Aquila Drone [13]' and 'Loon Balloon [14]' for implementation to provide broadband connectivity to the remote and rural ground users. However, their approach is quite monotonic and complex in deployment and usability under some specific and multiple network congestion scenarios.

Until now, there have been some proposals and ideas on future telecom generation business modelling and innovation in the literature. A new business model based on HetNet infrastructure sharing for maximizing users' data rate and creating more revenue to the operators was presented in [15], wherein the owners of infrastructure and the operators are proposed to be different. The infrastructure sharing can be a passive or active sharing method. The passive sharing involves sharing of the passive elements of the network like physical space, rooftops, towers, masts, etc. and backhaul connections by sharing the sites in a larger geographical area. The active sharing proposes to share the active infrastructure involving antennas, BSs, radio access networks and the core networks. 
Authors in [16] present that sharing increases the investment from the wireless service providers involved through some revenue-sharing contracts. According to their study, for the capacity sharing between the SPs, the incentive is greater if the owner of the infrastructure gets the larger fraction of the revenue carrying the overflow traffic.

A cloud assisted business model for small cell deployment was discussed in [17], that works by setting small cells to be in open access mode by the users or third parties for potential monetary rewards from the network operators, thereby allowing better service delivery to the customers of the network. Some business models based on the roles played by a Service Provider, as an Asset Provider (AP), Connectivity Provider (CP) and Partner Service Provider (PSP) were discussed in [18].

The authors in [19] have discussed a business model framework which is purely value-based, called as the 'V4 model', for the mobile network operators that is formed of four dimensions, namely Value Proposition, Value Network, Value Architecture, and Value Finance. They present a strong need by the network operators to enhance their ability in determining what constitutes the most viable business model, essential to meet their strategic objectives in the rapidly changing communication scenarios in their paper.

According to the discussion made in [20], making one single communications network that is capable of delivering all services across multiple industries and applications is very challenging, as every use case or application will need to fulfil different requirements. Their paper presents a study on the limitations of the current communications standards (3GPP LTE-Advanced, etc.) that fail to support the low-latency applications and hence put forward the urge to propose a whole new set of an innovative business model for the $5 \mathrm{G}$ future communications.

From past few years, there has been this growing research and interest in applying persuasive technology approach to business models to obtain desired behaviours [21]. The Fogg's [22] theoretical view on defining persuasive technology:

'A technology that is designed to change attitudes or behaviours of the users through persuasion and social influence, but not through coercion.'

Though the concept of persuasive technology approach can be applied to a number of fields, lately the focus has been into development and innovation of business models to a world of 5G communications [23]. The association of persuasive technologies to $5 \mathrm{G}$ business models are expected to bring better business to the network operators. The persuasive business model will try to attach to anything, anybody, anywhere and anytime, with the overall aim to 
'persuade', such that anything, anybody, anytime in any place will be linked persuasively together [24].

Lindgren et al. in their recent paper mentioned that the persuasive technology has digital components embedded and is situated within two dimensions combining knowledge and technology. The first dimension contains knowledge about how people and things can be triggered to change behaviour and the second dimension contains the technology, in which the ICT is in some way embedded and can communicate with the human, thing, or both in combination [25].

Another dimension to business modelling is to make use of disruptive technology. The disruption creates opportunities that are almost always associated with new products and services [26]. Although there has to be a positively associated risk-taking with the extent of disruptive Business Model Innovation (BMI) adoption.

A Business Model Cube was presented in [27], that discusses seven dimensions of business modelling namely value proposition, user and/or customer, value chain functions, competence, network, relations and value formulae. Our approach presents a perspective of Persuasive and Disruptive Business Modelling for the aerial radio architecture utilizing these seven dimensions and is discussed in detail in Section 4.1.

\section{Network Deployment Paradigm Shift}

In Section 1.2, we have mentioned the problem related to the PTC that needs attention by the service operators for the future wireless communications. We have discussed that how the existing network deployments might become inadequate in delivering the required services to the end users. In our previous papers, we have proposed a concept of an alternate cellular network system of 'Self-Itinerant Intelligent Aerial Radio Architecture (SIIARA) [5]' that is designed to follow the mass moving users. The SIIARA has been proposed as a complete and independently functioning cellular network that provides the cellular services to the ground users by deploying multiple ad-hoc networks of aerial base stations (Hovering Ad-Hoc Network (HANET) [7]), at the targeted and congested network areas. The members of the HANET can be arranged into different configurations as teams to offload the overloaded macro sites in a congestion affected network areas due to mass accumulated and moving users.

The architecture of SIIARA (see, Figure 1) proposed by us is a moving cellular network system that can follow the users to provide the required 
high-bandwidth data services on the move. This characteristic of SIIARA eliminates the need for adding fixed small cells required at the problematic network areas. Hence, the repeated need for an additional network infrastructure is mitigated.

We have investigated the SINR performance of the users, and the overall network under PTC affected network conditions on the deployment of SIIARA and improvements were observed in the SINR and user throughputs [28, 5] through the alternate deployment of the aerial cellular network at the target areas. We have further proposed and evaluated our concept of 'Distributed Dynamic Backhauling of the Aerial Architecture (DDBAA) [29]', and it was found that by dynamically linking the aerial backhaul network to the main ground network, the PTC problem can be solved to greater extent.

Through one of the aerial configurations, we have also investigated the performance of the HANETs into Aerial-HetNets (AHs) [30], by placing the individual aerial members at multiple altitude levels, showcasing the functionality and operability of ground HetNets from the space. We have scrutinized as to how AHs can be deployed to offload the ground sites and improve user SINRs by utilizing the $n=1$ reuse factor, thereby saving the spectrum.

Until now the network deployment paradigm followed by the service operators was to keep adding cell sites (low-power base stations) wherever the requirement arose. However, the network densification through small cell sites come with some challenges that might become worse with increasing number of subscribers in the future. The challenges and issues pertaining to site costs, acquisition of appropriate land/space, additional spectrum requirements, interference management, mobility management, and backhaul network congestion become crucial to resolving for the service operators.

The network infrastructure for the future wireless radio networks will follow the classic tower infrastructure business model, but we believe that a network deployment paradigm shift in the traditional network infrastructure is needed to accommodate the challenges and issues (mentioned above) for the $5 \mathrm{G}$ onwards $(5 \mathrm{G}+)$ radio systems. Not that the whole existing network infrastructure is required to change, but an alternate solution that can well assist and complement the available infrastructure especially during the 'Need \& Demand' situations.

Figure 2, depicts the network deployment paradigm shift for a typical PTC affected network scenario. The Figure represents mass user groups moving in a particular direction creating a need for service at the different time instants $\left(\mathrm{t}_{1}, \mathrm{t}_{2} \ldots \mathrm{t}_{\mathrm{n}}-1, \mathrm{t}_{\mathrm{n}}\right)$. 
224 P. L. Mehta et al.

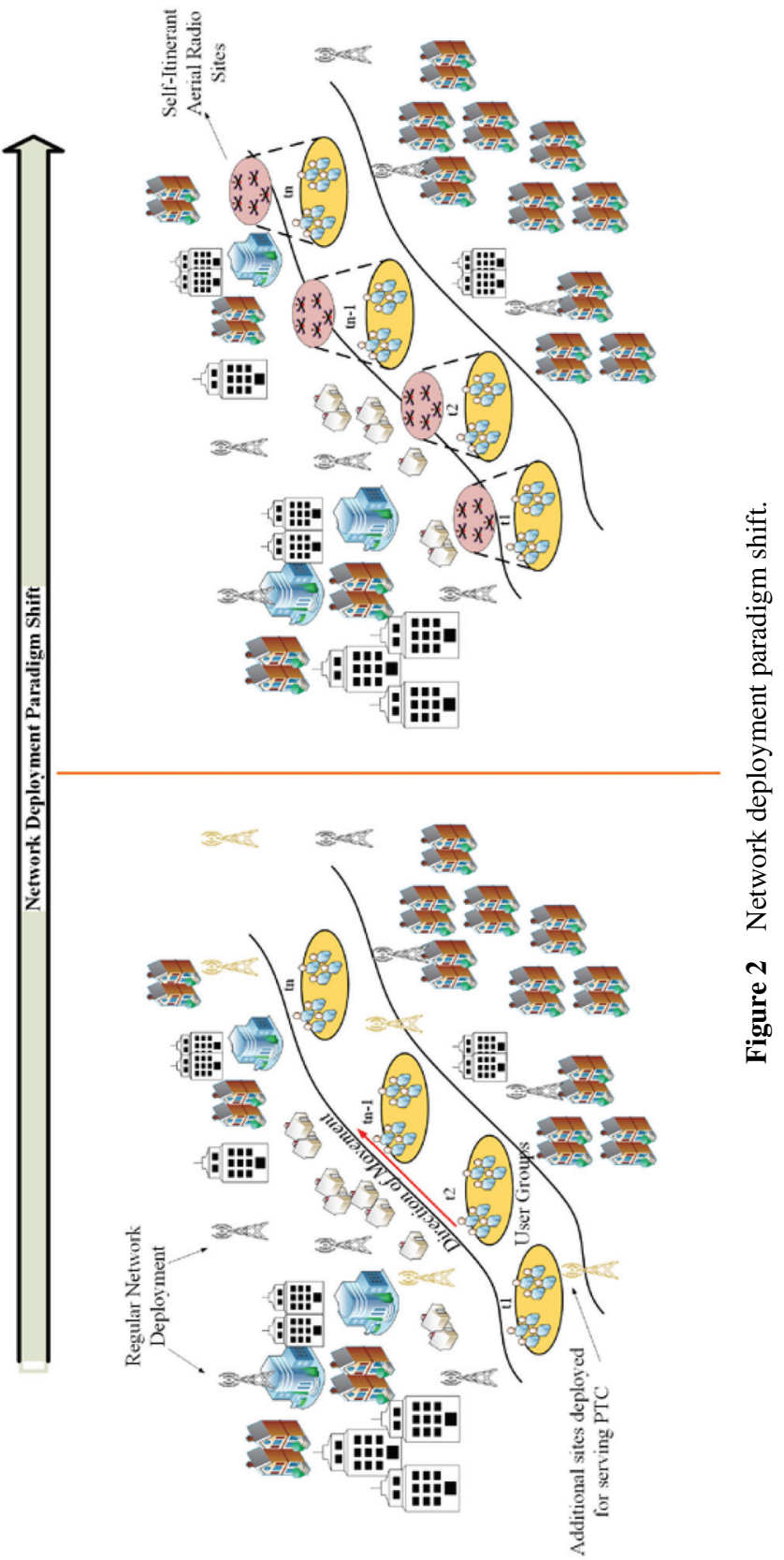


The first case illustrates the addition of fixed sites deployed to fulfil the service demands of the PTC causing users. This is the traditional business modelling case where sites are deployed wherever the demand is expected to be arising. However, the limit to the number of sites with increasing number of users is uncertain. The other case illustrates providing an alternate solution of aerial base stations hovering above the PTC causing users that carry the responsibility to follow the users and provide the required cellular services. This alternate solution clearly prevents the need to add more sites (that might be underutilized when the PTC problem dissipates) giving the liberty to reutilize the same aerial sites at other places and times to solve the PTC problem appearing at some other locations. Hence, we believe a place-time variant solution is suitable in servicing a place-time variant network problem.

\section{The Pragmatism: The Need to Propose an Innovative Business Model}

The traditional business modelling was centralized in providing a solution to single problems, with a limited and restrictive usage. Such business solutions cannot be extended to other applications and in multiple use-case scenarios. User Mobility is a dynamic pattern, and the network usages by such users change with time and place. The Service Providers need to meet this everincreasing demand for data while retaining profitability to their businesses. Hence, we believe that a single and conventional infrastructure solution will not suffice. We follow the Persuasive \& Disruptive Business Modelling (PDBM) approach in a pragmatic sense that will put forward the need for persuasion of our proposed concept and disrupt the concept of single and traditional business modelling approach implemented so far.

\subsection{The Pseudo-Disruptive Model Approach}

In Figure 2, we can see that just like any other new technology/concept, our unique idea of itinerant communication infrastructure, tends to disrupt the aucourant network architecture and deployment paradigm. Here the network paradigm that we are talking about is the network deployment process. Although the process pretends to be quite structured and definitive, it holds a certain degree of complexity and parameters to be incorporated. This includes the technology in use, the area morphology in which the network is deployed, the kind of users, and so on. All these parameters vigorously change with time and place, indicating the inevitability of variations in the network design 
and deployment. However, being an extremely expensive deal, the network deployments tend to follow a standard network process overlooking the local aberrations. This generic approach is a very averaged out, and often, the Network Service Providers (NSPs) pay the price by dealing with neverending hookups with a customer complaining about call drops and poor signal quality.

Figure 3 shows how and why an SIIARA can be a disruptive dimension of the business modelling of a network deployment. Figure 3 shows a sub-architecture of SIIRA that follows a densely packed user groups traversing a path. Here the itinerant aerial architecture disrupts the conventional way of serving users of deploying additional network sites to cover the path in use by flying above the serving the users beneath the SIIARA network layer (see, Figure 3). However, being a need-based architecture, the SIIARA network can co-exist with the conventional deployment business model and save the enormous expenses from draining out. The NSPs can become capable of serving the absurd and abrupt situations within the network area by promptly deploying the SIIARA architecture and revoking any additional network deployment. On the other hand, under certain cases, SIIARA might completely overhaul the conventional method

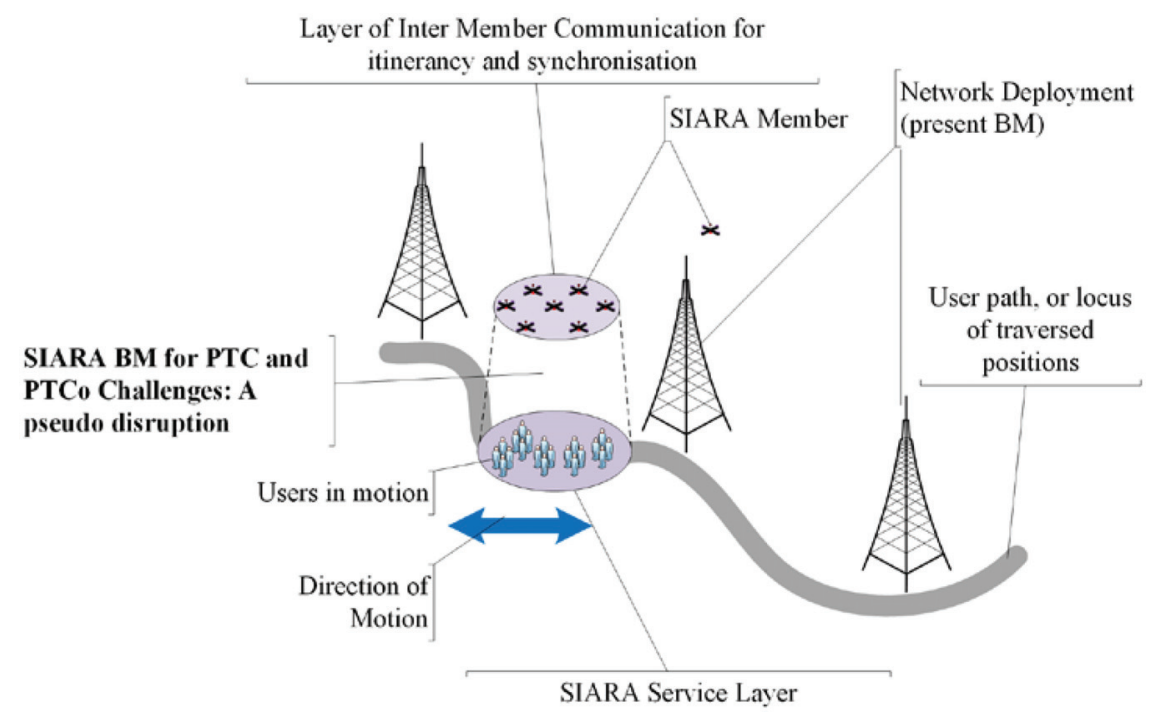

Figure 3 The Pseudo-disruptive and persuasive model approach. 
of network deployment, giving rise to a unique pseudo-disruptive and persuasive approach. The Section 4.2 and 4.3 shall discuss the coexistence and pragmatic business approach and the corresponding BMs in detail.

\subsection{The Pragmatic Penchant}

The pragmatism to our business modelling approach lies with the fact that we cannot eliminate or disrupt the traditional models completely, but to build an innovative model over it. Hence, we try to propose business model innovation through an agreement between the Persuasive and Disruptive business technology. We say our business approach is pragmatic because we believe it is a better approach to solve the issues concerning the future network scenarios, still involving the previous classic models. Therefore, we attempt to promote a smoother transition from the old models to the new models by building and innovating our concept. We attempt to provide business applications and solutions that help in maintaining the user satisfaction criteria and are still economically viable to the service operators, product manufacturers, stakeholders and all other third parties involved.

Through our proposed Aerial Radio Architectures, we aim to combat multiple challenges through a single solution. We present parallel business models for multiple network scenarios. We believe that the chances of product failure are minimal as it can be re-used in various applications. It is inevitable to use the Aerial Base Stations for the commercial and civilian use in the field of wireless communications. In this section, we will present business applications of the Aerial Radio Architecture that will give an understanding and a realistic vision to right business modelling and revenue fetching opportunities through different network case scenarios.

We propose and present the business case scenarios by employing the Persuasive and Disruptive business modelling approach.

\subsection{The Business Case Scenario}

We have used the term 'Pragmatic Business' to acknowledge the versatility that precipitates in the SIIARA Business Models (SBMs). As shown in Figure 4, there are several areas where the SBMs can be applied. Figure 5 shows the legends with respect to Figure 4. This gives an ample sturdiness in terms of unique selling points and the return of investments. From the BM-cube point of view [27], following presents the SBM analysis: 


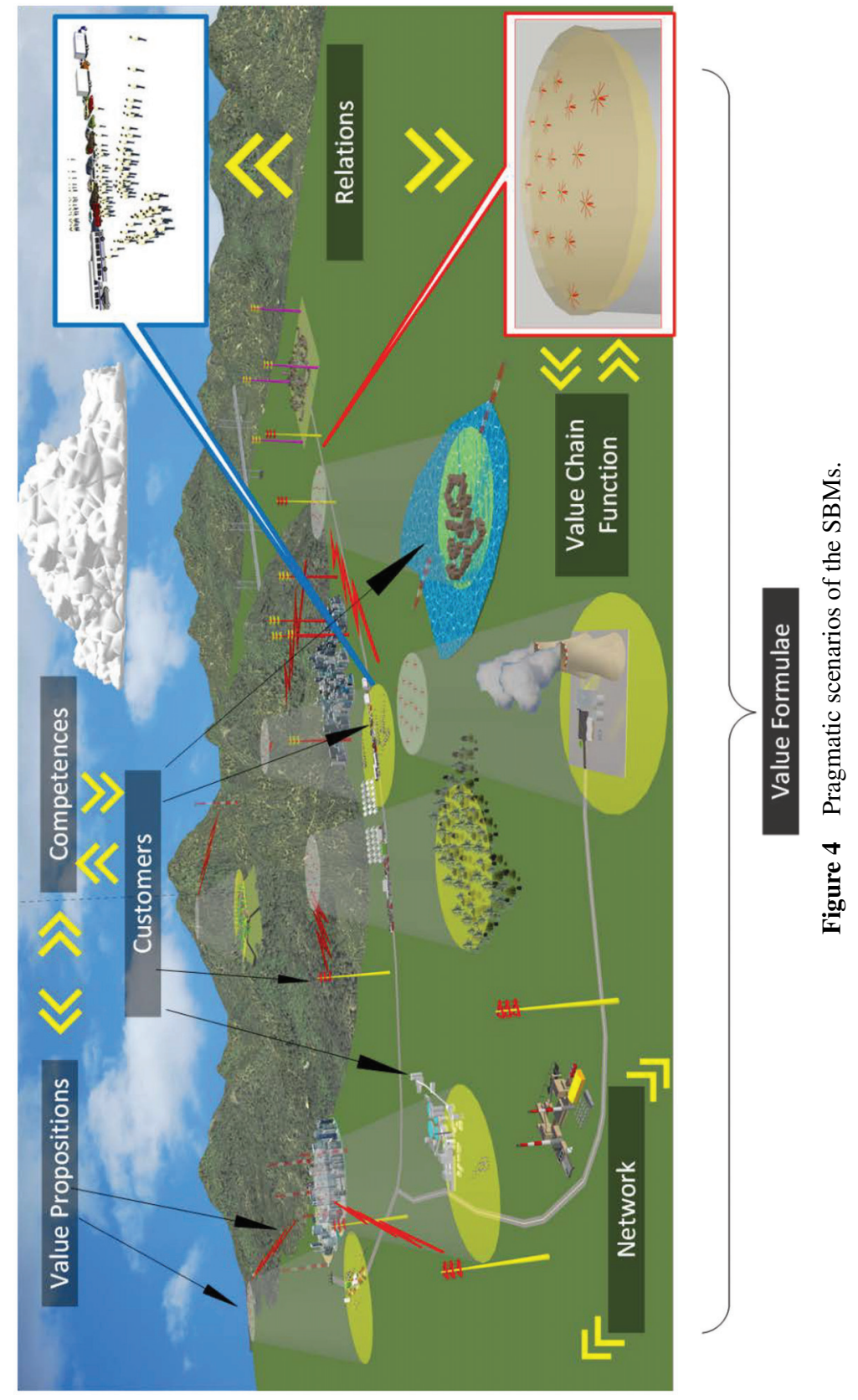




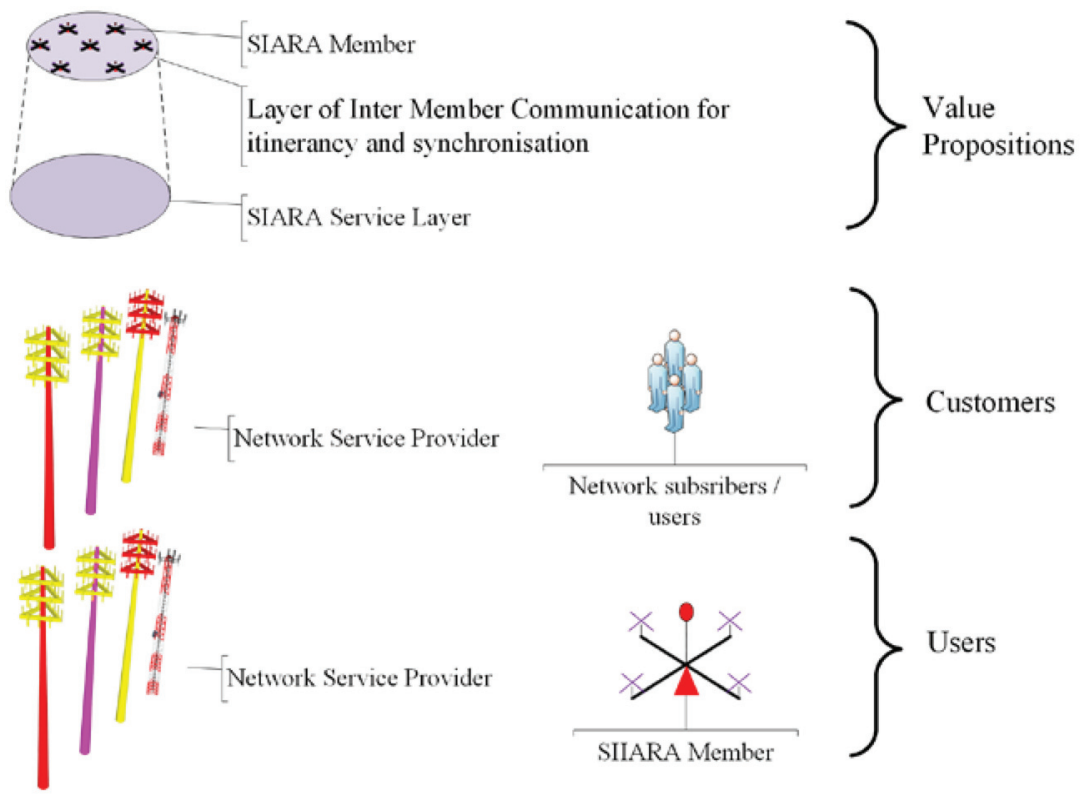

Figure 5 Legends of SBMs.

(a) Value Propositions: In Figure 5, we see an SIIARA sub-architecture (refer, Figure 1) as the 'value proposition' legend of Figure 4. Though, the SIIARA concept deals with the Place Time Coverage and Capacity $\left(\mathrm{PTC}^{2}\right)$ [31] challenge, its sub-architecture pitches a situation based value propositions across its deployment. The inter-member communication layer is a unique concept where several UAVs, which are termed as 'Members' in the SIIARA corresponding to aerial base station devices, can communicate with each other to form a 'constellation' of Members in serving the 'network situation' underneath. These situations eventually shape up as values of the SBM. Figure 4 shows the following major values an SIIARA architecture presents to the business domain:

i. Uplifting the throughput and coverage in an area where they are degraded due to an unprecedented accumulation of the potential PTC causing users. In the cities, the SIIARA service layer (see, Figure 3) acts as an alternate network that can well substantiate to be a boon to the primary network base stations choked from time to time, saving the overall expenses on the additional site deployment. 
ii. Serving users in the areas where the primary terrestrial network is absent or not possible to deploy. Some of the business case examples are mines, chemical and nuclear power plants, forests, etc. Under these situations, the SIIARA service layer (see, Figure 4) acts as primary service network to the underlying users.

iii. Providing emergency network service in areas where the existing primary network has been damaged due to natural or man-made calamities. The occurrence of flood or war-zones is two of such examples. Under such cases, the SIIARA service layer (see, Figure 4) acts as network extension of the primary network.

iv. Monitoring and surveillance at the borders or selected areas such as paddy fields. For such cases, the SIIARA service layer has a different purpose other than serving the mobile users. Here, the underneath scenario is more prioritized than user accumulations. The application is more intelligent and strategic than usual network services. Here, the intelligent member management is also a value proposition, as the efficient inter-member communication layer (see, Figure 3) that leads to efficient strategic applications.

(b) Customers and Users: As per the definition, the customers are those who pay with money and users are those who pay through service or other kinds [22]. In SBMs, the Client, people, and the SIIARA Member dealers are the either user, or customer, or both. Figure 5 shows the entities of SIIARA in users and customers categories.

i. By client, we mean NSPs, Defence, or Government Organisations. A client can both be user or customer, meaning that the SIIARA service either can be directly paid or can be awarded for the people's welfare, etc.

ii. By 'people' we mean both, the network subscribers, and the strategic entities, such as border trespassers or calamity sufferers. The network subscribers are essentially customers in the SBM as they are those who pay for the service and are mostly unaware of the system deployed for the services. Strategic entities are usually users as they provide business to SBM but are not paying directly.

iii. Member dealers are those who are manufacturing and maintaining the UAVs and Members (UAV with mounted technology radios and intelligent system).

(c) Network: No business is an island (Håkonsson 1990), and SBM is no exception. Figure 6. shows the network of the SBM in a very concise 
form. These networks when work in the chorus, shall make the SBM to work as expressed in Figure 4. Most of the networks, as shown in Figure 6, are physical in nature.

(d) Competencies: Figure 6 shows the inevitable competencies that we propose, an SBM should have, ranging from core to inherited competencies. In the Figure, we can see the associated network partners that SBM may have for its substantiality. Following are the competencies of SBM in detail:

i. Core Competence: The SBM's core competence shall be the
a. Network Design,
b. Installation and Integration,
c. Operations and Maintenance,
d. Sales and Marketing,
e. Human resource development,
f. Business Model Innovation,
g. Business culture.

that are in proximity with the service sector.

ii. Inherited Competences: There shall be other competence tributaries that the SBM may accommodate from network partners, such as
a. Research and Development,
b. Standardisation and IPR,
c. Supply chain,
d. Metallurgy,
e. Material engineering,
f. Foundry and fabrication,
g. Design and development, etc.

(e) Value Chain Functions: The value chain functions are functions which are able to "offer" value propositions and serve the customers and users with values [22]. Figure 6 shows the value chain functions associated to SBM. Through SIIARA, we propose an extremely sophisticated vehicle that has capability carry a payload for a considerable long duration subject to extreme capabilities of manoeuvring and sustainability. Therefore, for SIIARA to be in reality, the chain starts from mining and material extraction to development, distribution, and finally to service. Referring to Figure 6, the SBM value chains are:

i. Inbound logistics

ii. Operations, 
iii. Out bound logistics,

iv. Marketing and sales,

v. Service,

vi. Procurement,

vii. Human resource management,

viii. Administration and finance infrastructure,

ix. Business model innovation.

(f) Relations: SBM endorses on the strong relations with other businesses. Both Figures 4 and 6 show the strong inter-dependency of the various businesses.

i. Tangible relation: As we can see in Figure 6, certain other businesses, such as research and development, mining (material supply) and manufacturing have a very tangible relation with the SBM. Such networks have a direct impact on the design, development, upgradation and application of the SBM.

ii. Non-tangible relation: Sales, marketing and customer relations are the non-tangible, however, shall have a wider impression on the overall SBM.

(g) Value formulae: Through Figure 4, we have attempted to give an insight of the values that SBM offers. All the members that shall be deployed are monetarily related to the users/customers it will be serving. Each of the value propositions shall lead to a new value formula, alternate network deployment and strategic deployments are some of the examples.

Perspective: Apart from the 7-business dimensions discussed above, radical and conservative approaches are also the point of view to analyse a business model.

a. Radical: The SIIARA is a very novel and rigorous concept, and requires an entirely different temperament for its business modelling. Ingesting an entirely non-conventional approach of having UAV technology for the deployment of network base station is indeed a radical BM outfit.

b. Conservative: It can be seen in Figure 4 that members of the SIIARA form a constellation to serve the underneath situation. Each of these constellations has layers of commutations to perform the service and backhauling. We expect SIIARA to solve a magnitude of wireless network issues. However, we do not write-off the need of the terrestrial network. In fact, we propose SIIARA and terrestrial network work in coordination and sync for more rigorous and robust solutions. 


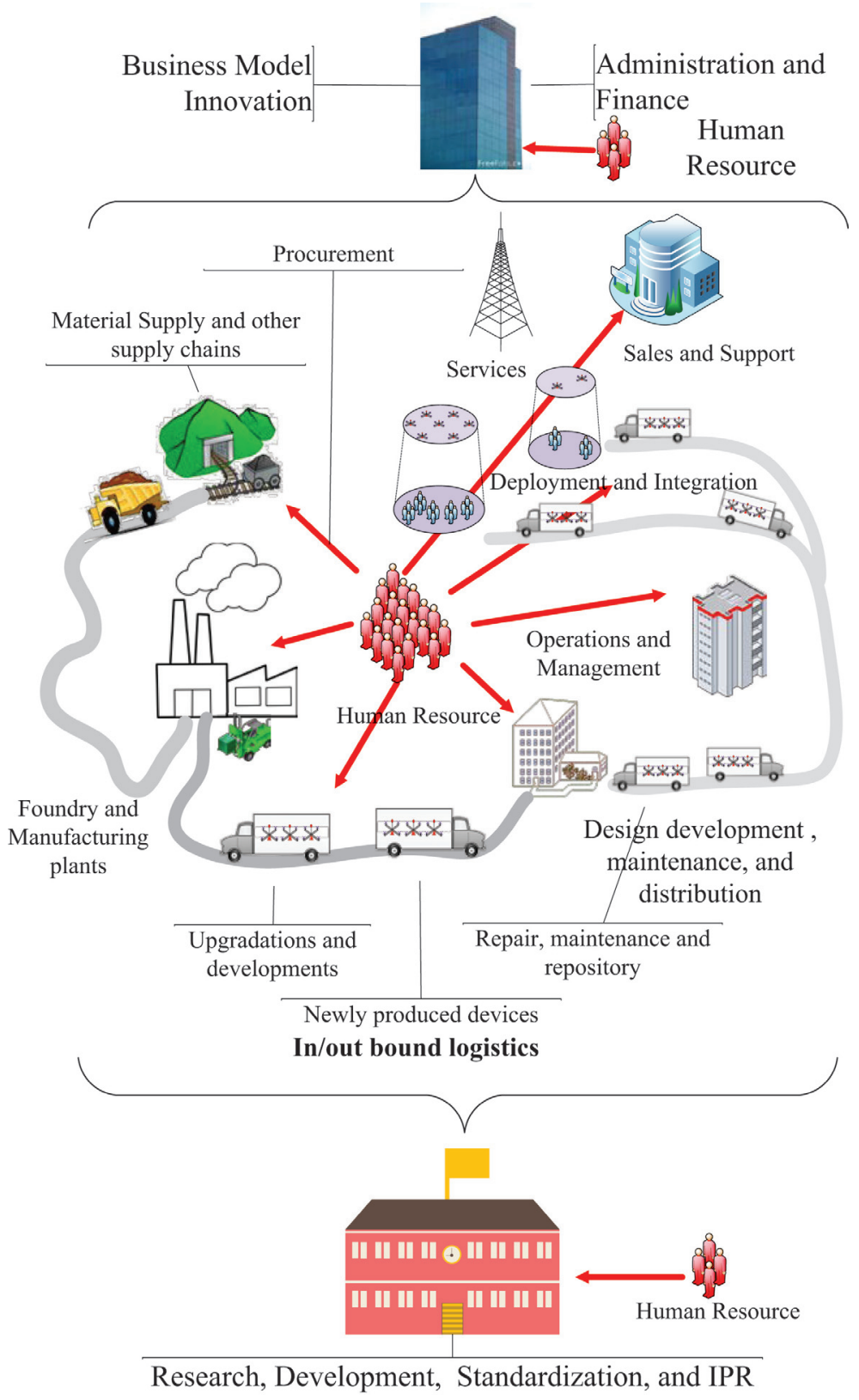

Figure 6 Competence, network, relations and value chain functions in SBM. 


\section{Conclusion and Future Work}

In this paper attempted to endorse the business perspective of a novel airborne communication architecture, which was technically investigated in our previous works. The novel architecture was termed previously by us as the Self Itinerant Intelligent Aerial Radio Architecture (SIIARA). The need of doing so was to estimate the functioning of a quantitatively researched concept in the business domain. This will give a comprehensive investigation of a technical research work performed by us. This paper is not only the extension of previous work but also opens a new avenue of business researches of technology. Here, we presented SIIARA Business Model (SBM) and dissected it in the seven BM-cube dimensions, where each dimension was thoroughly discussed from the business point of view. We have optimistically shown that the novel SIIARA architecture must not campaign with any definite approach. We have shown that SIIARA is a proponent of either side of business modelling, being either disruptive or persuasive, either radical or conservative, numerous value propositions but under one umbrella, interdependent but mutually sustainable value chains, and so on. Hence, we cannot tag the SBM with any of present BM kind, rather, we suggest incorporating all possible Business Dimensions in tuning them all together to form an aspect we termed as 'Pragmatic Business Approach. The pragmatic business approach, discussed in Section 4.2 leads to enormous opportunities that can be factorised into several business models and business cases. Each value propositions opens a pathway for future works.

\section{References}

[1] "Nokia_Ultra_Dense_Network_White_Paper_EN.pdf."

[2] Soldani, D., Pentikousis, K., Tafazolli, R., and Franceschini, D. (2014). 5G networks: End-to-end architecture and infrastructure [Guest Editorial]. IEEE Commun. Mag., 52(11), 62-64.

[3] "5G-Nework-Architecture-Whitepaper-en.pdf."

[4] Venkataraman, H., and Trestian, R. (Eds.). (2017). 5G Radio Access Networks: centralized RAN, cloud-RAN and virtualization of small cells. CRC Press.

[5] Mehta, P. L., Sørensen, T. B., and Prasad, R. (2016). "A Self-Itinerant Aerial Radio Architecture For Serving Place Time Variant User Accumulations," presented at the Wireless World Research Forum 2016. 
[6] Kumar, A., Mehta, P. L., and Prasad, R. (2014). Place Time CapacityA novel concept for defining challenges in $5 \mathrm{G}$ networks and beyond in India. In IEEE Global Conference on Wireless Computing and Networking (GCWCN), 278-282.

[7] Mehta, P. L., Sørensen, T. B., and Prasad, R. (2015). HANET: Millimeter wave based intelligent radio architecture for serving place time capacity issue. In 5th International Conference on Wireless Communications, Vehicular Technology, Information Theory and Aerospace \& Electronic Systems (VITAE) Wireless VITAE. IEEE Press. Hyderabad, India.

[8] "Mobile cell sites," Wikipedia. 29-Mar-2017.

[9] Jaziri, A., Nasri, R., and Chahed, T. (2016). Offloading traffic hotspots using moving small cells. In IEEE International Conference on Communications (ICC), 1-6.

[10] "Literature Review: The Business Model Concept and Its Use." [Online]. Available at: https://www.researchgate.net/publication/311998656_The Business_Model_Concept _and_Its_Use

[11] Teece, D. J. (2010). Business models, business strategy and innovation. Long Range Planning, 43, 172-194.

[12] Huawei, “"5G: A Technology Vision,' White paper,” Feb. 2014.

[13] "Flying Aquila: Early lessons from the first full-scale test flight and the path ahead," Facebook Code. [Online]. Available at: https://code.facebook.com/posts/268598690180189

[14] "Project Loon," Wikipedia. 17-Feb-2017.

[15] Meddour, D. E., Rasheed, T., and Gourhant, Y. (2011). On the role of infrastructure sharing for mobile network operators in emerging markets. Computer Networks, 55(7), 1576-1591.

[16] Berry, R., Honig, M., Nguyen, T., Subramanian, V., Zhou, H., and Vohra, R. (2013). On the nature of revenue-sharing contracts to incentivize spectrum-sharing. In Proceedings of the INFOCOM, 845-853.

[17] Zhang, N., Cheng, N., Gamage, A. T., Zhang, K., Mark, J. W., and Shen, X. (2015). Cloud assisted HetNets toward 5G wireless networks. IEEE Communications Magazine, 53(6), 59-65.

[18] S. D., and M. A, (2014). "A 5G Infrastructure for," J. Telecommun. Syst. Manag., 1-10.

[19] Al-Debei, M. M., and Avison, D. (2011). Business model requirements and challenges in the mobile telecommunication sector. Journal of Organisational Transformation \& Social Change, 8(2), 215-235.

[20] Lema, M. A., et al., (2017). Business case and technology analysis for 5G low latency applications. IEEE Access, 5, 5917-5935. 
[21] "RP_Journal_2245-456X_121.pdf"

[22] "Persuasive Technology - 1st Edition." [Online]. Available at: https://www.elsevier.com/books/persuasive-technology/fogg/978-155860-643-2

[23] Lindgren, P. (2016). "Multi Business Model Innovations Towards 2050 and Beyond," in Wireless World in 2050 and Beyond: A Window into the Future!, R. Prasad and S. Dixit, Eds. Springer International Publishing, 149-160.

[24] Lindgren, P. (2016). Multi Business Model Innovation in a World of 5G: What Will Persuasive Business Models Look Like in a World of 5G? Wireless Personal Communications, 88(1), 79-84.

[25] "Secure Persuasive Business Models and Business Model Innovation in a World of 5G |SpringerLink." [Online]. Available at: https://link-springercom.zorac.aub.aau.dk/article/10.1007/s11277-017-4101-y

[26] Karimi, J., and Walter, Z. (2016). Corporate entrepreneurship, disruptive business model innovation adoption, and its performance: The case of the newspaper industry. Long Range Plann., 49(3), 342-360.

[27] Lindgren, P., and Rasmussen, O. H. (2013). The business model cube. Journal of Multi Business Model Innovation and Technology, 1(3), 135-180.

[28] Mehta, P. L., Sørensen T. B., and Prasad, R. (2016). SINR based capacity performance analysis of hovering ad-hoc network. In 19th International Symposium on Wireless Personal Multimedia Communications (WPMC), 147-152.

[29] Mehta, L. P., Sørensen, T. B., and Prasad, R. (2016). Distributed Dynamic Backhauling in Self-Itinerant Intelligent Aerial Radio Architecture. In Global Wireless Summit (GWS).

[30] Mehta, P. L., and Prasad, R. (2017). Aerial-Heterogeneous Network: A Case Study Analysis on the Network Performance Under Heavy User Accumulations. Wireless Personal Communications, 96(3), 3765-3784.

[31] Kumar, A. (2016). “Active Probing Feedback based Self Configurable Intelligent Distributed Antenna System: For Relative and Intuitive Coverage and Capacity Predictions for Proactive Spectrum Sensing and Management," PhD Dissertation, Aalborg Universitet, Aalborg. 


\section{Biographies}

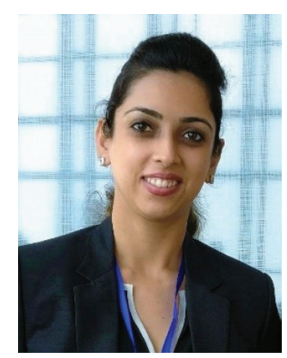

Purnima Lala Mehta, Assistant Professor, Electronics and Communication Department at HMR Institute of Technology and Management, Delhi, India, has successfully defended her PhD research entitled "SELF ITINERANT INTELLIGENT AERIAL RADIO ARCHITECTURE (SIIARA) For coverage and capacity enhancement" in April 2018. The degree is awarded by the Department of Business Development and Technology, School of Business and Social Sciences, Aarhus University, Herning, Denmark. She has done most of her technical research from the Department of Electronic Systems, Aalborg University, Denmark. Prior to her PhD research, she has received her MTech (ECE) degree from NorthCap University (formerly ITM University), Gurgaon, India, and B.E (E\&TC) from University of Pune, Bharati Vidyapeeth's College of Engineering from Women, Pune, India.

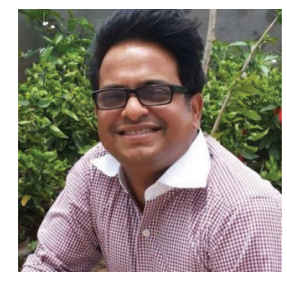

Ambuj Kumar received Bachelor of Engineering in Electronics \& Communications from Birla Institute of Technology, India in the year 2000. He undertook Internship Training at the Institut für Hochfrequenztechnik, Technical University, (RWTH, Germany). He had worked at the Lucent Technologies Hindustan Private Limited (India) and with leading service providers namely Vodafone on the Mobile Radio Network Design; Macro and Microcell planning; Optimization for the GSM and the CDMA-based Mobile Communication Networks; the green field deployments; and planning, deployment, 
optimization of rapidly expanding GSM and Edge networks across India. He has worked as Research Associate at the Centre for TeleInFrastruktur (CTIF), Department of Electronic Systems, Aalborg University (Denmark). Ambuj Kumar was awarded scholarship under European Commission programme for doing $\mathrm{PhD}$ in Aalborg University. Ambuj Kumar had also worked at the Vihaan Networks Limited (India), he developed facilities for experimental studies on 'Advanced Alternative Networks'. He had worked as Research Assistant in the eWall Project, funded by the European Commission. He was awarded Doctor of Philosophy in December 2016 by the Aalborg University, on his thesis "Active Probing Feedback Based Self Configurable Intelligent Distributed Antenna System For Relative and Intuitive Coverage and Capacity Predictions for Proactive Spectrum Sensing and Management". Currently, Dr. Kumar is working as PostDoc in the Department of Business Development and Technology, Aarhus University (Denmark) since February, 2017. His research interests are radio wave propagation, cognitive radio, visible light communications, and radio resource management etc. He has more than 10 research publications including a book chapter in thematic areas.

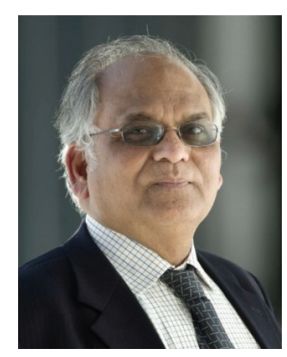

Ramjee Prasad, Fellow IEEE, IET, IETE, and WWRF, is a Professor of Future Technologies for Business Ecosystem Innovation (FT4BI) in the Department of Business Development and Technology, Aarhus University, Herning, Denmark. He is the Founder President of the CTIF Global Capsule (CGC). He is also the Founder Chairman of the Global ICT Standardisation Forum for India, established in 2009. GISFI has the purpose of increasing of the collaboration between European, Indian, Japanese, North-American and other worldwide standardization activities in the area of Information and Communication Technology (ICT) and related application areas.

He has been honored by the University of Rome "Tor Vergata", Italy as a Distinguished Professor of the Department of Clinical Sciences and Translational Medicine on March 15, 2016. He is Honorary Professor of 
University of Cape Town, South Africa, and University of KwaZulu-Natal, South Africa.

He has received Ridderkorset af Dannebrogordenen (Knight of the Dannebrog) in 2010 from the Danish Queen for the internationalization of top-class telecommunication research and education.

He has received several international awards such as: IEEE Communications Society Wireless Communications Technical Committee Recognition Award in 2003 for making contribution in the field of "Personal, Wireless and Mobile Systems and Networks", Telenor's Research Award in 2005 for impressive merits, both academic and organizational within the field of wireless and personal communication, 2014 IEEE AESS Outstanding Organizational Leadership Award for: "Organizational Leadership in developing and globalizing the CTIF (Center for TeleInFrastruktur) Research Network", and so on.

He has been Project Coordinator of several EC projects namely, MAGNET, MAGNET Beyond, eWALL and so on.

He has published more than 40 books, 1000 plus journal and conference publications, more than 15 patents, over $100 \mathrm{PhD}$ Graduates and larger number of Masters (over 250). Several of his students are today worldwide telecommunication leaders themselves. 
